\title{
It's not over until it's over: the chameleonic behavior of COVID-19 over a six-day period
}

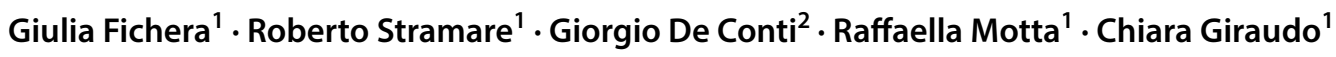

Received: 6 April 2020 / Accepted: 16 April 2020 / Published online: 29 April 2020

(C) Italian Society of Medical Radiology 2020

\begin{abstract}
The current global outbreak of COVID-19 represents a major challenge in terms of epidemiology, contagiousness, treatment, as well as clinical and radiological behavior of this disease. Radiological imaging plays a key role in the diagnostic process and during the monitoring of the clinical conditions especially of patients with severe symptoms. According to the preliminary data collected in our tertiary center, we have documented a peculiar behavior in patients requiring endotracheal intubation who underwent seriate chest X-rays. In fact, the radiological pattern of COVID-19 patients may worsen despite a prompt amelioration after the onset of mechanical ventilation. Thus, according to our initial evidence, we recommend to perform seriate chest $\mathrm{X}$-rays in the days following the onset of mechanical ventilation even if the immediate monitoring suggests an improvement. Studies on a larger scale are necessary to fully assess the findings at chest radiographs of critical, mechanically ventilated patients and their correlation with the long-term outcome.
\end{abstract}

Keywords COVID-19 $\cdot$ Radiology $\cdot$ Chest X-ray $\cdot$ Outbreak

\section{Introduction}

The current global outbreak of COVID-19 represents a major challenge in terms of epidemiology, contagiousness, treatment, as well as clinical and radiological behavior of this disease [1]. Radiological imaging plays a key role in the diagnostic process and during the monitoring of the clinical conditions especially of patients in the intensive care unit (ICU) [2]. In particular, using computed tomography (CT) not only the extension and the severity of the disease can be precisely characterized, but according to the recent study of Bai et al. [3], even the distinction between COVID-19 and other viral pneumonia can be performed.

It has also been shown that in patients who are not in need of mechanical ventilation $\mathrm{CT}$ abnormalities increase within 10 days and that between 5 and 8 days after the onset of the symptoms the infection rapidly aggravates $[4,5]$.

Chiara Giraudo

chiara.giraudo@unipd.it

1 Department of Medicine - DIMED, Institute of Radiology, University of Padova, Via Giustiniani 2, 35100 Padova, Italy

2 Radiology Unit, Azienda Ospedaliera di Padova, Padova, Italy
Nevertheless, it should be highlighted that patients with COVID-19 hospitalized in the ICU and receiving mechanical ventilation are usually monitored with chest X-rays not only because the transportation to the radiology unit might be extremely time consuming, could worsen the clinical conditions of the patients, and may cause disease spread but also because of the higher radiation exposure associated with multiple CT scans.

Although there is a paucity of information regarding the role of chest radiographs in patients with COVID-19, radiologists should become familiar with the characterization of the disease at traditional imaging.

According to preliminary data collected in our tertiary center, we have documented a peculiar behavior of the disease in patients requiring endotracheal intubation who underwent seriate chest X-rays. Indeed, we have observed that among 18 patients ( 16 males and 2 females; mean age \pm standard deviation of $66.8 \pm 13.5$ years) undergoing tracheal intubation within $72 \mathrm{~h}$ from hospital admission, ten showed a prompt (within $24 \mathrm{~h}$ ) improvement of the radiological findings at chest X-ray, six remained stable, and two showed a progression of the disease (Figs. 1 and 2). Nevertheless, in the following days, over an interval of 6 days from intubation, four patients previously stable or with a former improvement (overall 33.3\%) had a rapid worsening of the 

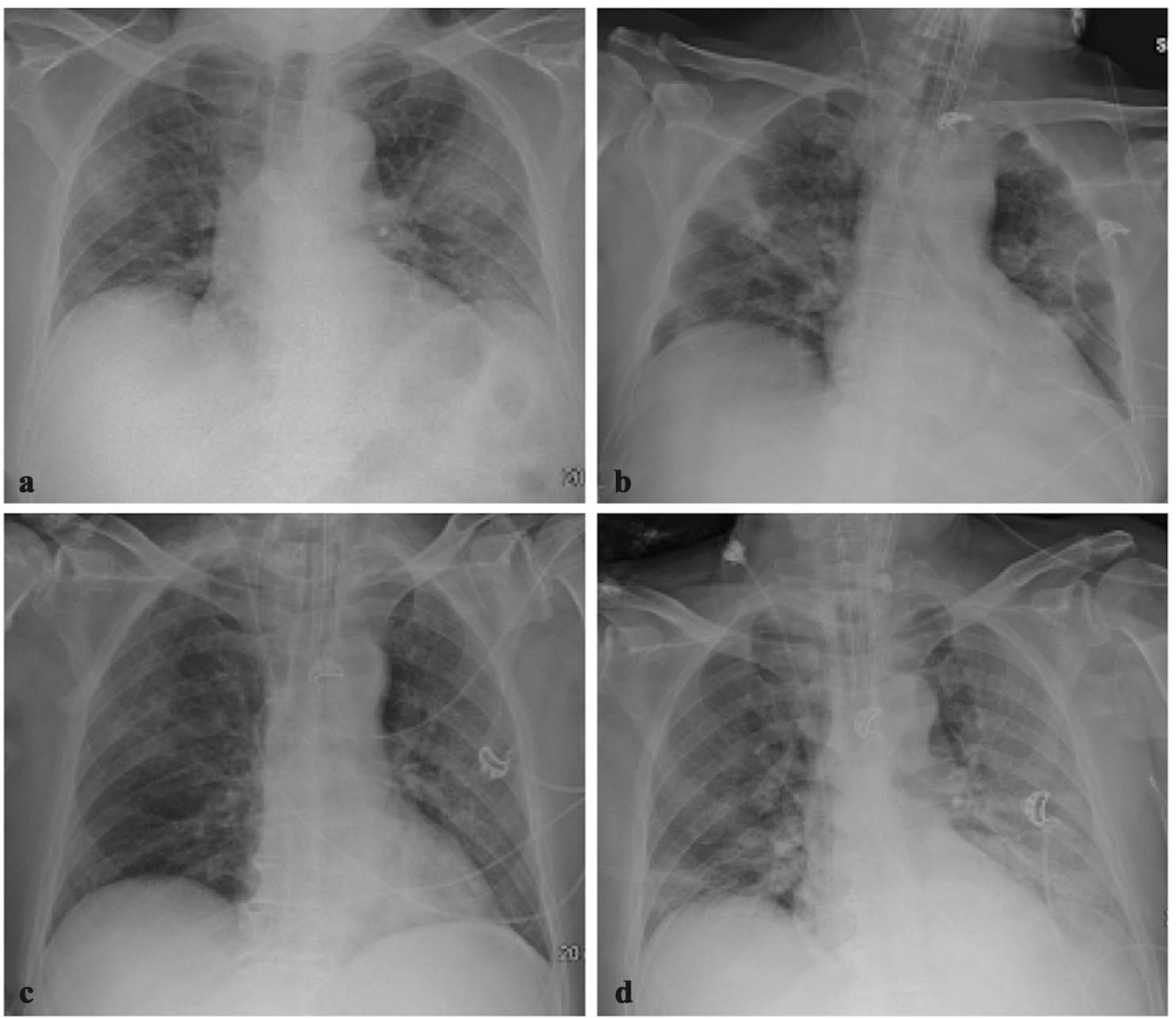

Fig. 1 Chest X-rays of a 73-year-old male patient affected by COVID-19 (a-d). The first chest radiograph at admission demonstrated bilateral pulmonary perihilar consolidations (a). Due to a worsening of the clinical conditions, during the second day after admission, he underwent endotracheal intubation (b) with a significant improvement of the radiological findings within $24 \mathrm{~h} \mathrm{(c).} 72 \mathrm{~h}$ later, the radiological findings worsened again especially in the left lung (d)
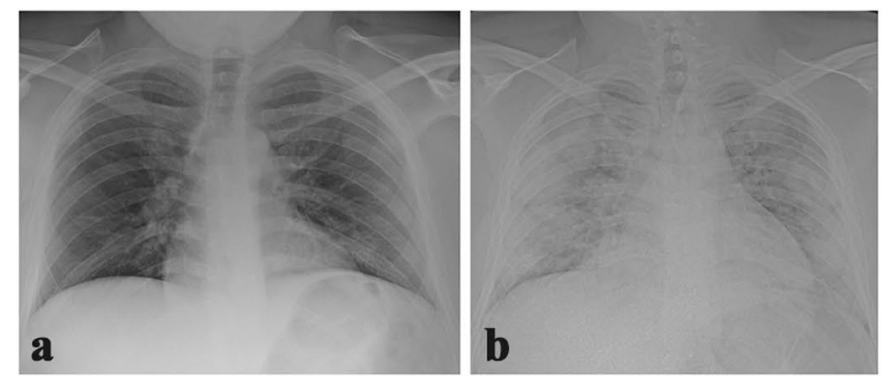

Fig. 2 Chest X-rays of a 27-year-old male patient with COVID-19 (a-d). The first radiograph at admission demonstrated a mild interstitial thickening in the lower lobes (a). Within $72 \mathrm{~h}$ from hospital admission, his clinical conditions worsened and bilateral pulmonary consolidations became visible at chest $\mathrm{X}$-ray (b). The same day he
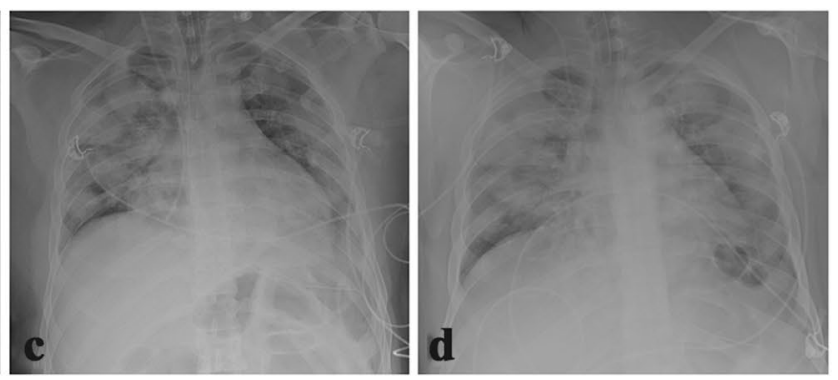

was intubated with a prompt improvement of the radiological findings especially in the left lung (c). In the next $24 \mathrm{~h}$ after the beginning of the mechanical ventilation, the bilateral pulmonary consolidations increased (d) 
clinical conditions; four patients $(22.3 \%)$ remained stable and eight progressively improved (44.4\%). At the chest $\mathrm{X}$-ray performed at admission, nine showed an interstitial pattern and eight pulmonary consolidations. Only one had a negative chest X-ray which then evolved within $24 \mathrm{~h}$ in consolidations. Within 6 days, $89 \%$ of the patients $(n=16)$ showed a wide patch of parenchymal consolidations at imaging, a finding which is consistent with the literature [3, 4]. One patient died 18 days after hospital admission.

Our results suggest a progression of the disease despite a temporary improvement or a short interval of stable conditions after intubation. Thus, according to our preliminary evidence, we recommend to perform seriate chest X-rays in the days following the onset of mechanical ventilation even if the immediate monitoring suggests a radiological amelioration of the pattern.

Several studies already demonstrated the chameleonic and heterogeneous behavior of COVID-19 infections [6]. For instance, Shi et al. [7] reported early changes at CT in asymptomatic patients whereas Xie et al. [8] in their study focused on CT identified pathologic findings at radiological imaging despite initial negative RT-PCR tests. Thus, CT also taking advantage of low-dose protocols certainly plays a pivotal role not only in detecting subtle initial findings, but it also accurately characterizes severe cases [9].

Chest X-ray, on the contrary, surely has a limited role in diagnosing the most common initial pattern of COVID-19 typically defined by ground glass. Nevertheless, we should not overlook the importance of this tool in clinical practice, especially when dealing with hospitalized patients in ICU affected by severe infections with pulmonary consolidations.

In fact, traditional imaging can guarantee a reliable continuous monitoring of ICU patients and guide therapeutic decisions.

In the province of Hubei, the introduction of CT changes among the diagnostic criteria for COVID-19 led to an improvement in the diagnostic performance [5]. We can speculate that for hospitalized patients in the ICU chest $\mathrm{X}$-rays may represent one of the main tools for clinical management.

We call for further international, multicentric studies describing the longitudinal evolution of the disease at imaging, considering gender, age, nationality and comorbidities, which may help us in defining regular and specific timing for $\mathrm{CT}$ scans and better defining the role of chest X-ray.

Studies on a larger scale are necessary to fully assess the findings at chest radiographs of critical, mechanically ventilated patients and their correlation with the long-term outcome.
Authors contribution All authors have approved the manuscript and have significantly contributed to it.

\section{Compliance with ethical standards}

Conflict of interest None of the authors has any conflict of interest to declare.

Ethical approval All procedures in studies involving human participants were in accordance with the ethical standards of the institutional and/or national research committee and with the 1964 Declaration of Helsinki and its later amendments or comparable ethical standards.

Informed consent For this type of study, formal consent is not required.

Consent for publication For this type of study, consent for publication is not required.

\section{References}

1. Kim H (2020) Outbreak of novel coronavirus (COVID-19): What is the role of radiologists? Eur Radiol. https://doi.org/10.1007/ s00330-020-06748-2

2. Giovagnoni A (2020) Facing the COVID-19 emergency: we can and we do. Radiol Med. https://doi.org/10.1007/s11547-02001178-y

3. Bai HX, Hsieh B, Xiong Z et al (2020) Performance of radiologists in differentiating COVID-19 from viral pneumonia on chest CT. Radiology. https://doi.org/10.1148/radiol.2020200823

4. Pan F, Ye T, Sun P et al (2020) Time course of lung changes on chest CT during recovery from 2019 novel coronavirus (COVID19) pneumonia. Radiology. https://doi.org/10.1148/radiol.20202 00370

5. Pan Y, Guan H, Zhou S et al (2020) Initial CT findings and temporal changes in patients with the novel coronavirus pneumonia (2019-nCoV): a study of 63 patients in Wuhan. Eur Radiol, China. https://doi.org/10.1007/s00330-020-06731-x

6. Lee EYP, Ng MY, Khong PL (2020) COVID-19 pneumonia: What has CT taught us? Lancet Infect Dis. https://doi.org/10.1016/ S1473-3099(20)30134-1

7. Shi H, Han X, Jiang N et al (2020) Radiological findings from 81 patients with COVID-19 pneumonia in Wuhan, China: a descriptive study. Lancet Infect Dis. https://doi.org/10.1016/S1473 -3099(20)30086-4

8. Xie X, Zhong Z, Zhao W, Zheng C, Wang F, Liu J (2020) Chest CT for typical 2019-nCoV pneumonia: relationship to negative RT-PCR testing. Radiology. https://doi.org/10.1148/radiol.20202 00343

9. Agostini A, Floridi C, Borgheresi A et al (2020) Proposal of a low-dose, long-pitch, dual-source chest CT protocol on thirdgeneration dual-source $\mathrm{CT}$ using a tin filter for spectral shaping at $100 \mathrm{kVp}$ for Corona Virus Disease 2019 (COVID-19) patients: a feasibility study. Radiol Med. https://doi.org/10.1007/s1154 7-020-01179-x

Publisher's Note Springer Nature remains neutral with regard to jurisdictional claims in published maps and institutional affiliations. 